\title{
Association between Quality of Sleep and Chronic Periodontitis: A Case-control Study
}

\author{
Prabhu Subramani ${ }^{1}$, Vishnumaya Ajikumar ${ }^{2}$
}

\begin{abstract}
Background: Periodontitis is a ubiquitous chronic inflammatory disease affecting the supporting structures of the teeth, and if not promptly diagnosed and correctly managed can ultimately lead to tooth loss. Sleep is a complex and essentially biological process that is required on a daily basis for all humans regardless of age, sex, or ethnic origin. Since inflammation is characteristic of both chronic periodontitis and sleep deprivation, the present study was undertaken to study the relationship between quality of sleep and chronic periodontitis.

Materials and methods: A case-control study was conducted among the outpatients reporting to Asan Memorial Dental College and Hospital. The sample size for the study was $n=198$. The survey comprises of demographic data of study participants followed by an interviewer administered questionnaire to assess the quality of sleep using the Pittsburgh sleep assessment scale followed by clinical assessment to record periodontal health status using community periodontal index (CPI) by WHO.

Results: Patients who were sleep deprived showed increased loss of attachment from 3 to $5 \mathrm{~mm}$ to more than $12 \mathrm{~mm}$. Odds ratio showed that patients with periodontitis had 3.36 times the risk of sleep deprivation compared to patients without periodontitis.

Conclusion: Lack of good quality of sleep will be a risk factor for periodontitis.

Keywords: Periodontitis, Quality of sleep, Sleep.

Indian Journal of Sleep Medicine (2019): 10.5005/jp-journals-10069-0040
\end{abstract}

\section{INTRODUCTION}

Periodontitis is a ubiquitous chronic inflammatory disease affecting the supporting structures of the teeth, and if not promptly diagnosed and correctly managed can ultimately lead to tooth loss. They are inflammatory diseases in which microbial etiologic factors induce a series of host responses that mediate inflammatory events. A number of risk factors contribute to the susceptibility of individuals to periodontal diseases, and to the pathogenesis and severity of the disease. These factors include smoking, diabetes, immunosuppressant, genetic factors, stress, and age. ${ }^{1}$

Sleep is a complex and essentially biological process that is required on a daily basis for all humans regardless of age, sex, or ethnic origin. Sleep has important roles in controlling the factors of many other body systems, and this becomes very evident in states of sleep deprivation. ${ }^{2}$ Inadequate sleep has effects on learning, memory processing, the repair of cell damage, brain development, neurobehavioral performances, hormonal regulation, risk of depression, increased cortisol and ghrelin, impaired glucose metabolism, and increased inflammatory and pro-inflammatory markers. ${ }^{3}$

Both acute and chronic sleep deprivations may activate inflammatory processes, leading to increasing $C$ reactive protein concentrations, increased peripheral circulation of leukocytes, and increased levels of interleukin- 6 and tumor-necrosis factor alpha. ${ }^{2}$ Higher interleukin- 6 levels were associated with lower quality of sleep among healthy asymptomatic men and women and increases the risk of destructive periodontal disease. Tumor-necrosis factor alpha is one of the key periodontal pathogens induced in destructive periodontal disease. It promotes the destruction of alveolar bone by stimulating the formation of osteoclasts and regulates matrix metalloproteases which are capable of degrading connective tissue. ${ }^{4}$

Since inflammation is characteristic of both chronic periodontitis and sleep deprivation, the present case-control study was
1,2Department of Public Health Dentistry, Asan Memorial Dental College and Hospital, Chengalpettu, Tamil Nadu, India

Corresponding Author: Prabhu Subramani, Department of Public Health Dentistry, Asan Memorial Dental College and Hospital, Chengalpettu, Tamil Nadu, India, Phone: +91 9080756119 , e-mail: prabhu.dent@gmail.com

How to cite this article: Subramani P, Ajikumar V. Association between Quality of Sleep and Chronic Periodontitis: A Case-control Study. Indian J Sleep Med 2019;14(3):43-45.

Source of support: Nil

Conflict of interest: None

undertaken to study the association between quality of sleep and chronic periodontitis.

\section{Materials and Methods}

A case-control study was conducted among the outpatients reporting to Asan Memorial Dental College and Hospital. Prior to the start of the study, approval was obtained from the Institutional Scientific Review Board, Asan Memorial Dental College and Hospital. The sample size for the study was $n=198$ based on the study conducted by Singh et al., calculated using G Power Statistical software. ${ }^{2}$ Patients aged $>18$ years with no history of systemic diseases diagnosed with generalized chronic periodontitis were included in the case group and patients without periodontitis age and gender matched were taken into the control group. Patients undergoing periodontal therapy and patients with systemic illness like diabetes, taking medications which can have an influence on periodontium were excluded.

Following collection of demographic data of study participants, an interviewer administered questionnaire, the Pittsburgh sleep 
quality index (PSQI), ${ }^{5}$ which was used to assess the quality of sleep. It differentiates "poor" from "good" sleep quality by measuring seven areas (components): subjective sleep quality, sleep latency, sleep duration, habitual sleep efficiency, sleep disturbances, use of sleeping medications, and daytime dysfunction over the last month. A total score of " 5 " or greater is indicative of poor sleep quality. Periodontal health status was assessed using mouth mirror and $\mathrm{CPI}$ probe, and CPI was recorded as per WHO. ${ }^{6}$ Three indicators of periodontal status are used for this assessment: gingival bleeding, calculus, and periodontal pockets.

The mouth was divided into sextants defined by tooth numbers: 18-14, 13-23, 24-28, 38-34, 33-43, and 44-48. A sextant was examined only if there were two or more teeth present, which was not indicated for extraction.

Index teeth: for adults aged 20 years and over, the teeth examined were the following:

\begin{tabular}{lllll}
\hline 17 & 16 & 11 & 26 & 27 \\
47 & 46 & 31 & 36 & 37 \\
\hline
\end{tabular}

The two molars in each posterior sextant are paired for recording, and if one is missing, there was no replacement. If no index teeth or tooth is present in a sextant qualifying for examination, all the remaining teeth in that sextant were examined and the highest score was recorded as the score for the sextant. Distal surfaces of third molars were not scored.

For subjects under the age of 20 years, only six index teeth-16, $11,26,36,31$, and 46 were examined. When children under the age of 15 years are examined, pockets were not recorded, i.e., only bleeding and calculus were considered.

Examination and recording: the index teeth or all remaining teeth in a sextant where there is no index tooth were probed and the highest score was recorded in the appropriate box. The codes were the following:

0 -healthy

1-bleeding observed, directly or by using a mouth mirror, after probing.

2-calculus detected during probing, but all of the black band on the probe visible.

3-pocket 4-5 $\mathrm{mm}$ (gingival margin within the black band on the probe).

4 -pocket $6 \mathrm{~mm}$ or more (black band on the probe not visible).

$\mathrm{X}$-excluded sextant (less than two teeth present)

9-not recorded

\section{Loss of Attachment}

The extent of loss of attachment was recorded using the following codes:

0 -loss of attachment $0-3 \mathrm{~mm}$ (cemento enamel junction (CEJ) not visible and CPI score $0-3$ )

1-loss of attachment 4-5 mm (CEJ within the black band).

2-loss of attachment 6-8 $\mathrm{mm}$ (CEJ between the upper limit of the black band and the $8.5-\mathrm{mm}$ ring).

3-loss of attachment 9-11 $\mathrm{mm}$ (CEJ between the 8.5- $\mathrm{mm}$ and $11.5-\mathrm{mm}$ rings).

4-loss of attachment $12 \mathrm{~mm}$ or more (CEJ beyond the $11.5-\mathrm{mm}$ ring).

$\mathrm{X}$-excluded sextant (less than two teeth present).

9-not recorded (CEJ neither visible nor detectable).
A single examiner performed clinical examination to assess the periodontal health status. The examiner was trained and calibrated in the Department of Public Health Dentistry, Asan Memorial Dental College and Hospital and the intraexaminer reliability was $K=0.86$. Data were entered in Microsoft excel and analyzed using SPSS Version 23. Odds was used to assess the strength of association between quality of sleep and chronic periodontitis.

\section{Results}

Gingivitis and periodontitis were commonly encountered in dental practice; periodontal pathogens are found to be risk factors in cardiac problems, diabetes, preterm birth, etc., the present study was undertaken to test the association between periodontitis and quality of sleep. Figure 1 depicts the distribution of study subjects according to gender and periodontitis; among the $n=198$ study subjects, $n=107$ were male of which $n=52$ (26.3\%) had periodontitis and $n=55$ (27.8\%) were free from periodontitis; $n=91$ were female of which $n=47$ (23.7\%) had periodontitis and $n=44$ $(22.2 \%)$ are free from periodontitis, and there is no difference in the prevalence of periodontitis across gender $(p>0.05)$.

Table 1 depicts the CPI score among the study subjects according to sleep score, bleeding on probing and presence of calculus were more among the study subjects with PSQI score $>5$, and the difference is not statistically significant $(p>0.05)$

Table 2 depicts the loss of attachment score among the study subjects according to pattern of sleep, loss of attachment was more among the study subjects with high PSQI score compared to the subjects with less PSQI score; however, the difference is not statistically significant $(p>0.05)$.

Table 3 depicts the association between PSQI score and periodontitis among the study subjects, $n=80(40.4 \%)$ study subjects had PSQI score $>5$ of which $n=54(67.5 \%)$ had periodontitis and $n=26$ (32.5\%) were free from periodontitis; $n=118(59.6 \%)$ study subjects had PSQI score $<5$ of which $n=45$ (38.1\%) had periodontitis and $n=73$ (61.9\%) were free from periodontitis. Chi-square test for association showed that there is a significant association between pattern of sleep and periodontitis $(p<0.05)$. Odds ratio showed that patients with periodontitis had 3.36 times the risk of sleep deprivation compared to patients without periodontitis.

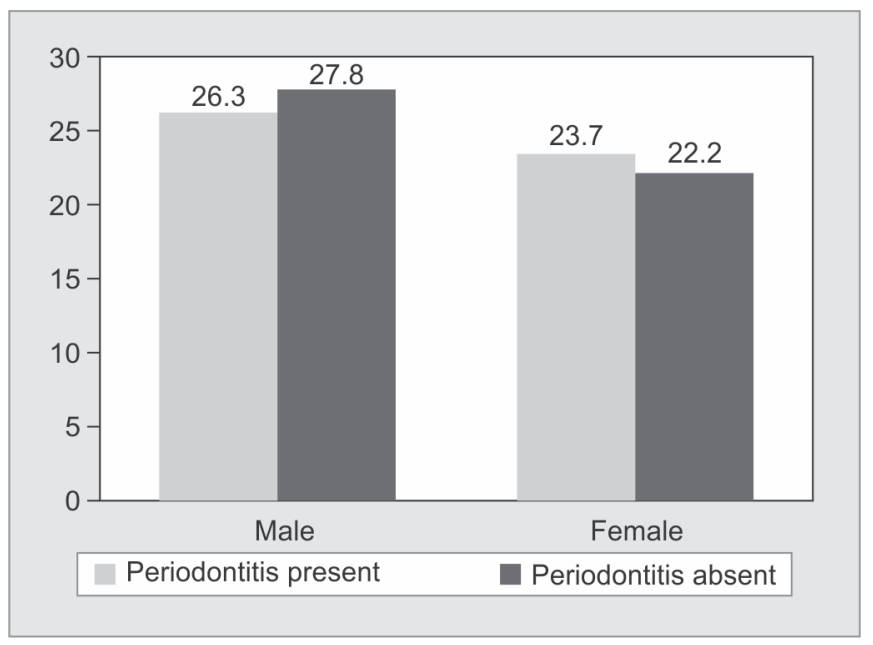

Fig. 1: Distribution of study subjects according to gender and prevalence of periodontitis 
Table 1: Community periodontal index score among the study subjects

\begin{tabular}{|c|c|c|c|c|c|c|c|c|c|c|c|c|}
\hline \multirow[b]{2}{*}{ CPIscore ${ }^{*}$} & \multicolumn{2}{|c|}{ Healthy } & \multicolumn{2}{|c|}{ Bleeding } & \multicolumn{2}{|c|}{ Calculus } & \multicolumn{2}{|c|}{ Shallow pocket } & \multicolumn{2}{|c|}{ Deep pocket } & \multicolumn{2}{|c|}{ Total } \\
\hline & $n$ & $\%$ & $n$ & $\%$ & $n$ & $\%$ & $n$ & $\%$ & $n$ & $\%$ & $n$ & $\%$ \\
\hline Sleep affected* & 14 & 17 & 21 & 26.2 & 24 & 28.9 & 10 & 13 & 11 & 14.9 & 80 & 40.4 \\
\hline Sleep not affected* & 32 & 25.2 & 18 & 15.3 & 28 & 24.1 & 16 & 14.2 & 24 & 20.8 & 118 & 59.6 \\
\hline
\end{tabular}

${ }^{*}$ Chi-square test ( $p>0.05$-not significant)

Table 2: Loss of attachment score among the study subjects

\begin{tabular}{|c|c|c|c|c|c|c|c|c|c|c|c|c|}
\hline \multirow{2}{*}{$\begin{array}{l}\text { Loss of attachment } \\
\text { score* }\end{array}$} & \multicolumn{2}{|c|}{$0-0$ to $3 \mathrm{~mm}$} & \multicolumn{2}{|c|}{$1-4$ to $5 \mathrm{~mm}$} & \multicolumn{2}{|c|}{$2-6$ to $8 \mathrm{~mm}$} & \multicolumn{2}{|c|}{$3-9$ to $11 \mathrm{~mm}$} & \multicolumn{2}{|c|}{4 to $>12 \mathrm{~mm}$} & \multicolumn{2}{|c|}{ Total } \\
\hline & $n$ & $\%$ & $n$ & $\%$ & $N$ & $\%$ & $n$ & $\%$ & $n$ & $\%$ & $n$ & $\%$ \\
\hline Sleep affected* & 21 & 26.2 & 18 & 23.3 & 20 & 24.4 & 14 & 16.8 & 7 & 9.3 & 80 & 40.4 \\
\hline Sleep not affected* & 48 & 39.5 & 19 & 15.5 & 27 & 23 & 15 & 14.3 & 9 & 7.7 & 118 & 59.6 \\
\hline
\end{tabular}

${ }^{*}$ Chi-square test ( $p>0.05$-not significant)

Table 3: Comparison of Pittsburgh sleep quality index score and periodontitis

\begin{tabular}{|c|c|c|c|c|c|c|c|}
\hline \multirow[b]{2}{*}{ PSQI score* } & \multicolumn{2}{|c|}{ Periodontitis present* } & \multicolumn{2}{|c|}{ Periodontitis absent* } & \multirow[b]{2}{*}{ Chi-square value } & \multirow[b]{2}{*}{ Degrees of freedom } & \multirow[b]{2}{*}{$p$ value } \\
\hline & $n$ & $\%$ & $n$ & $\%$ & & & \\
\hline >5-sleep affected & 54 & 67.5 & 26 & 32.5 & 16.4 & 1 & 0.0014 \\
\hline$<5$-sleep not affected & 45 & 38.1 & 73 & 61.9 & & & \\
\hline
\end{tabular}

${ }^{*}$ Chi-square test $(p<0.05$ - statistically significant)

\section{Discussion}

Sleep deprivation is known to adversely affect cognitive and motor performances. This might impair an individual's capacity to perform adequate oral hygiene procedures, increasing the risk of periodontal disease. ${ }^{1}$

The biological plausibility for such potential association exists that quality of sleep interferes with the modulation of host immune and inflammatory responses. Researchers found an increase in lymphocytic activation with the overproduction of IL-1, IL-6, IL-7, and TNF alpha in sleep deprived individuals. ${ }^{7,8}$

The principal objective of the study was to analyze the association between the quality of sleep and periodontitis. The results in the present study are in agreement with study done by Singh et al., which stated a possible association between periodontal disease and inadequate sleep. ${ }^{2}$

When comparison was done with a previous study done by Ramoji Rao et al., ${ }^{9}$ periodontitis was present in male was $43.65 \%$ in the previous study and $26.3 \%$ in the current study. In female, $23.7 \%$ in the current study and $53.3 \%$ in the previous study. The reason being that the previous study was conducted among the general population and the current study was done among the outpatients reporting to Asan Dental College.

The individuals who were sleep affected with periodontitis was $67.5 \%$ in the current study and $56.75 \%$ in a study done by Singh et al., ${ }^{2}$ and the individuals who have sleep affected with periodontitis was $38.1 \%$ in the current study and $43.24 \%$ in the previous study. The reason being variation in the lifestyle patterns and habits among the different study subjects.

\section{LiMITATIONS}

The present study only includes a questionnaire to assess the quality of sleep. Various methods like electroencephalogram (EEG) and electromyogram (EMG) are not carried out in this study and further studies need to be undertaken with the above aids.

\section{CONCLUSION}

Within the limits of the present investigation, there appears to be a significant association between periodontal disease and sleep deprivation.

\section{References}

1. Grover V, Malhotra R, Kaur H. Exploring association between sleep deprivation and chronic periodontitis: a pilot study. J Indian Soc Periodontol 2015;19(3):304-307. DOI: 10.4103/0972-124X.154173.

2. Singh VP, Gan JY, Lei WL, et al. Association between quality of sleep and chronic periodontitis: a case-control study in Malaysian population. Dent Res J (Isfahan) 2019;16(1):29-35. DOI: 10.4103/17353327.249555.

3. Weiner RC. Relationship of routine inadequate sleep duration and periodontitis in a nationally representative sample. Sleep Disord 2016;2016:9158195. DOI: 10.1155/2016/9158195.

4. Khosravi R, Ka K, Huang T, etal.Tumornecrosis factor- $a$ and interleukin-6: potential interorgan inflammatory mediators contributing to destructive periodontal disease in obesity or metabolic syndrome. Mediators Inflamm 2013;2013:728987. DOI: 10.1155/2013/728987.

5. Buysee DJ, Reynolds 3rd CF, Monk TH, et al. The Pittsburgh sleep quality index: a new instrument for psychiatric practice and research. Psychiatry Res 1989;28(2):193-213. DOI: 10.1016/0165-1781(89)90047-4.

6. Oral Health Surveys - Basic Methods. Fifth Edition - WHO. Accessed from https://apps.who.int/iris/bitstream/handle/10665/97035/9789 241548649_eng.pdf;jsessionid=50A3576CB50A5EAA9188CA41E541 $B C 83$ ?sequence $=1$ on 5.05.2019.

7. Sauvet $F$, Leftheriotis $G$, Gomez-Merino D, et al. Effect of acute sleep deprivation on vascular function in healthy subjects. J Appl Physiol (1985) 2010;108(1):68-75. DOI: 10.1152/japplphysiol.00851.2009.

8. Frey DJ, Fleshner M, Wright Jr KP. The effects of 40 hours of total sleep deprivation on inflammatory markers in healthy young adults. Brain Behav Immun 2007;21(8):1050-1057. DOI: 10.1016/j.bbi.2007.04.003.

9. Ramoji Rao MV, Katari PK, Vegi L, et al. Prevalance of periodontal diseases among rural population of Mustabad, Krishna District. $J$ Int Soc Prev Community Dent 2016;6(Suppl 1):S59-S63. DOI: 10.4103/2231-0762.181169. 